\title{
Application of Evolutionary Multiobjective Algorithms for solving the problem of Energy Dispatch in Hydroelectric Power Plants
}

\author{
Carolina G. Marcelino ${ }^{1}$, Leonel M. Carvalho ${ }^{3}$, \\ Elizabeth F. Wanner ${ }^{2}$, Paulo E. M. Almeida ${ }^{1}$ and Vladimiro Miranda ${ }^{3}$ \\ ${ }^{1}$ Intelligent Systems Laboratory - LSI | CEFET MG - Belo Horizonte, Brasil \\ ${ }^{2}$ Computer Science Department - DECOM | CEFET MG - Belo Horizonte, Brasil \\ ${ }^{3}$ Centre for Power and Energy Systems - CPES | INESC TEC - Porto, Portugal \\ E-mails: (carolina, pema)@1si.cefetmg.br, efwanner@decom.cefetmg.br, \\ (lcarvalho, vmiranda)@inesctec.pt
}

\begin{abstract}
The Brazilian population increase and the purchase power growth have resulted in a widespread use of electric home appliances.Consequently, the demand for electricity has been growing steadily in an average of 5\% a year. In this country, electric demand is supplied predominantly by hydro power. Many of the power plants installed do not operate efficiently from water consumption point of view. Energy Dispatch is defined as the allocation of operational values to each turbine inside a power plant to meet some criteria defined by the power plant owner. In this context, an optimal scheduling criterion could be the provision of the greatest amount of electricity with the lowest possible water consumption, i.e. maximization of water use efficiency. Some power plant operators rely on "Normal Mode of Operation" (NMO) as Energy Dispatch criterion. This criterion consists in equally dividing power demand between available turbines regardless whether the allocation represents an efficient good operation point for each turbine. This work proposes a multiobjective approach to solve electric dispatch problem in which the objective functions considered are maximization of hydroelectric productivity function and minimization of the distance between NMO and "Optimized Control Mode" (OCM). Two well-known Multiobjective Evolutionary Algorithms are used to solve this problem. Practical results have shown water savings in the order of million $\mathrm{m}^{3} / \mathrm{s}$. In addition, statistical inference has revealed that NSGA-II algorithm is more robust than SPEA-II algorithm to solve this problem.
\end{abstract}

Key words: Multiobjective Optimization, NSGA-II, SPEA-II, Energy Efficiency.

\section{Introduction}

The demand for electricity is one of the key emerging issues in current energy management in Brazil. According to the annual report of Brazilian Energy Planning Company ("Empresa de Planejamento Energético" - EPE, in Portuguese), the share of renewable energy in electricity mix has dropped to $79.3 \%$ in 2013 due to unfavourable hydrological conditions despite owning the most diverse and extensive river networks from 
around the world. Consequently, thermal generation has taken over which has resulted in increased $\mathrm{CO}_{2}$ emissions and a less environmentally friendly generation mix. Fig. 1 shows a comparison between generation of electric power in Brazil in relation to the World and OECD member countries.

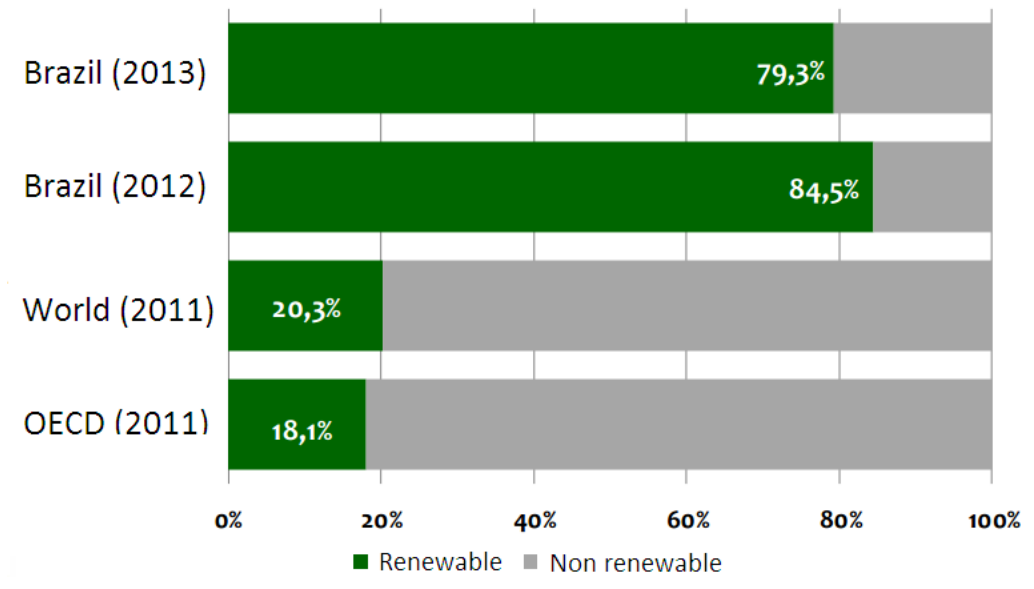

Fig. 1. Comparing energy generation matrix - 2013 (Source: EPE, Brazil)

This comparison reveals that Brazil has an advantage regarding the use of renewable energy over the rest of the World. Also notable is the reduction in renewable energy use between years of 2012 and 2013. This was caused by a widespread drought in 2013 causing thermal power plants to be called for production in order to maintain domestic demand for electricity. Hence, proper use of water resources is now an emerging topic in Brazil to guarantee that power system remains sustainable in future years.

The optimal scheduling of hydroelectric power plants (HPP), which are composed by several turbines, pipes turbines and connected electric generators or, in simple terms, generation units, is known as the hydro unit commitment problem. The objective consists in determining which generation units need to be on and their respective electric power set-point (in MW) so that overall hydroelectric power plant operation cost is minimized while meeting with the power required to be produced by the whole plant and satisfying the constraint set.

Electric power set-points are defined to each available generation unit at the hydroelectric plant, given some criteria to be met, such as operating limits, etc. This last problem, which is named as energy dispatch optimization problem, can only be solved if the production model for the whole hydroelectric plant is available. The majority of hydroelectric plants operators in Brazil equally distribute total power required to be produced by the plant among the available units. In this paper this will be referred as the "Normal Mode of Operation" (NMO). However, one cannot say that this simple dispatch criterion presents a good operation point for each unit since it does not take into account whether each unit will be operating close to its optimal operational point or not. The problem of finding optimal distribution of power demand among units of a power plant is complex, due to non-linearities of the productivity function and the high number of continuous and discrete constraints involved. 


\subsection{State of the Art}

Several optimization techniques to improve energy production efficiency in power systems were discussed in [1]. That study was motivated by the signing of Kyoto Protocol by European Union in 1997 which has led to the definition of 2020 climate and energy package commonly known as "20-20-20" targets. Accordingly, researchers have sought to find new methods and to use new optimization techniques to improve EU's energy efficiency in $20 \%$ by 2020 , which is one of the goals of that agreement. Some of the techniques described in [1] are: Search Algorithms, Evolutionary Algorithms, Simulated Annealing, Tabu Search, Ant Colony Optimization, Particle Swarm Optimization (PSO), Genetic Algorithms (GA) and Evolutionary Programming. Among them, GA is recommended to minimize energy losses and to maximize efficiency.

Baños [2] conducted a review of metaheuristic-based optimization techniques that have been applied so far to solve renewable energy optimization problems. The main conclusion of his survey is that the number of scientific papers that used metaheuristics to solve these problems has dramatically increased over the last few years. However, he has also reported that, in many cases, computational cost of using these methods is high even when using parallel processing techniques.

Finardi [3] proposed a new mathematical model for long-term planning of hydroelectric power plants. Linear programming was used to solve the problem of energy dispatch. This approach was shown to have a high computational cost which makes this model infeasible to be used for real time Energy Dispatch. Despite of the interesting results obtained, the author has not clearly discussed how important variables of the production function were discarded making the model very difficult to be understood and validated.

Abrao [4] proposed to use an artificial neural network to model the production function of a single generation unit. The author solved short-term planning problem, which consists on defining the respective dispatch of each generation unit for a specific period of time, using a version of Differential Evolution (DE) algorithm and a version of a PSO algorithm. However, computational cost of this solution is relatively high.

Marcelino [5] proposed a new mathematical model to solve HPP energy dispatch problem using DE. He showed that evolutionary strategy DE/best/1/bin is the most efficient for solving the mono-objective version of this problem. This model proved to be efficient and provided very promising results.

The research work reported in [6] shows the application and multiobjective algorithms to solve the classic electrical dispatch problem. The test case used, which is based on IEEE 30-bus system, comprises thermal and hydro units. Results indicate that SPEA algorithm achieved the best results compared to algorithms NSGA and NPGA, where the goals are to minimize carbon emissions and to minimize production cost.

Zhou [7] proposes a new multiobjective algorithm, which is named Multiple Group Search Optimizer (MGSO), to solve the classic electrical dispatch problem for IEEE-30 bus and IEEE 118-bus systems. The objectives are to minimize carbon emissions and production cost of power plants. Practical results of MSGO proved to be competitive when compared to results of NSGA-II and SPEA-II.

This work proposes a multiobjective approach to solve energy dispatch problem in HPP using a mathematical model very similar to the one proposed in [5]. For this 
purpose, two objective functions are defined: maximization of hydroelectric production function of whole HPP and minimization of distance between "Normal Mode of Operation" (NMO) and "Optimized Control Mode" (OCM). Note that OCM is the outcome of former objective. The latter objective is very interesting from practical point of view as HPP operators are not used to employ OCM and sometimes this mode of operation can be suspicious. As a case study, the proposed approach is applied to a large HPP operating in Brazil.

The paper is organized as follows: Section 2 describes the problem of Energy Dispatch in HPP; Section 3 presents the multiobjective problem proposed and algorithms used to solve it; Section 4 shows the case study, outcome of experiments and a simple comparative statistical analysis between different algorithms used; Section 5 presents final conclusions.

\section{Multiobjective Problem}

A multiobjective problem is characterized by having two or more objective functions, which are generally self conflicting. This type of problem does not have a single solution but a set of optimal solutions. A multi-objective optimization problem can be formulated as:

$$
\begin{aligned}
& \mathbf{x}^{*}=\min _{x} \mathbf{f}(\mathbf{x}) \\
& \text { subject to: }\left\{\begin{array}{l}
\mathbf{g}_{\mathbf{i}}(\mathbf{x}) \leq 0 ; i=1,2, \cdots, r \\
\mathbf{h}_{\mathbf{j}}(\mathbf{x})=0 ; j=1,2, \cdots, p
\end{array}\right.
\end{aligned}
$$

in which $\mathbf{x} \in \mathbb{R}^{n}, \mathbf{f}(\cdot): \mathbb{R}^{n} \rightarrow \mathbb{R}^{m}, \mathbf{g}(\cdot): \mathbb{R}^{n} \rightarrow \mathbb{R}^{r}$, and $\mathbf{h}(\cdot): \mathbb{R}^{n} \rightarrow \mathbb{R}^{p}$. Functions $\mathbf{g}_{\mathbf{i}}(\mathbf{x})$ and $\mathbf{h}_{\mathbf{j}}(\mathbf{x})$ are, respectively, inequality and equality constraints. Vectors $\mathbf{x} \in \mathbb{R}^{n}$ are called parameters of the multiobjective problem and belong to a parameter space. Vector function $\mathbf{f}(\mathbf{x}) \in \mathbb{R}^{m}$ belongs to a vectorial space called objective space.

In multobjective problems, there is not a single solution which is best or global optimum with respect to all objectives. Presence of multiple objectives in a problem usually gives rise to a family of non dominated solutions, called Pareto-optimal set, where each objective component of any solution along Pareto front can only be improved by degrading at least one of its other objective components.

Given two solutions, $\mathbf{x}$ and $\mathbf{y}$, it is said that $\mathbf{x}$ dominates $\mathbf{y}$ (denoted $\mathbf{x} \succeq \mathbf{y}$ ) if following conditions are met:

1. The solution $\mathbf{x}$ is at least equal to $\mathbf{y}$ for all objective functions;

2. The solution $\mathbf{x}$ is best than $\mathbf{y}$ for at least one objective.

\subsection{Evolutionary Multiobjective Algorithms}

Studies related to evolutionary multiobjective algorithms date back to 1980 s. The first algorithm of this class based on Pareto Front was proposed in early 1990s and is named as Multiobjective Genetic Algorithm (MOGA) [8]. After this, some other algorithms have emerged: Niched Pareto Genetic Algorithm (NPGA) [9], Nondominated Sorting Genetic Algorithm (NSGA) [10] (and its evolution NSGA-II [11]) and Strength Pareto 
Evolutionary Algorithm (SPEA) [12] (and its evolution SPEA-II [13]). Since then, several other evolutionary multiobjective algorithms were proposed and published. A general evolutionary multiobjective algorithm can be run by the following pseudocode:

Step 1. Initialize population;

Step 2. $\mathrm{Q}(\mathrm{t}=0)=q^{1} \ldots q^{\mu}$;

Step 3. Initialize population of archive $A(t=0)=0$;

Step 5. While ( $\rightarrow$ stop criteria) do

1. $\mathrm{P}(\mathrm{t}) \leftarrow \mathrm{Q}(\mathrm{t})$;

2. $\mathrm{S}(\mathrm{t}) \leftarrow$ selection $(\mathrm{P}(\mathrm{t}))$;

3. $\mathrm{R}(\mathrm{t}) \leftarrow$ crossover $(\mathrm{S}(\mathrm{t}))$;

4. $\mathrm{Q}(\mathrm{t}) \leftarrow$ mutation $(\mathrm{R}(\mathrm{t}))$;

5. $\mathrm{A}(\mathrm{t}) \leftarrow$ file update $(\mathrm{Q}(\mathrm{t}), \mathrm{A}(\mathrm{t}))$;

6. $\mathrm{t} \leftarrow \mathrm{t}+1$.

This paper uses well-known evolutionary multiobjective algorithms, NSGA-II and SPEA-II, to solve the proposed problem.

\section{The Problem of Electric Dispatch}

The electricity production of HPP is result of a process of potential and kinetic energy transformations. The potential energy stored in reservoir is transformed into mechanical energy by the turbine through its shaft, which, in turn, is transmitted to a electric generator unit. The electrical generator transforms mechanical energy into electrical energy. The power produced goes through collector electrical substation and is injected in transmission system to be delivered to consumption centres. A turbine-generator set has a specific hydraulic curve which characterizes its efficiency according to specific water flow and reservoir net head. This curve is called Hill Curve or Efficiency Curve, see Fig. 2.

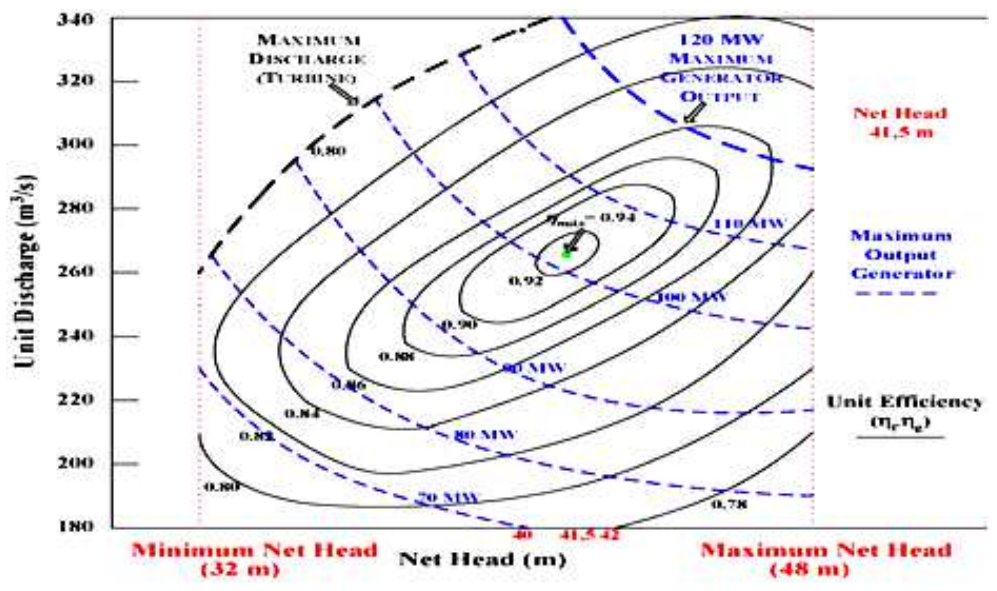

Fig. 2. Hill Curve example [5]. 
The efficiency curve contains important information to be considered when planning a HPP operation. Given this, it is possible to extract operating limits of turbinegenerator set, allowable range for net head in the dam, and also minimum and maximum points of efficiency, where the point of maximum efficiency is in the center of its contours.

From this, one can easily understand that the efficiency curve must be taken into account to ensure power generation with minimal use of water resources while considering operational constraints of a hydroelectric plant. This optimization problem can be characterized as the maximization of electricity production efficiency of whole HPP. In other words, the solution of this problem aims to generate more power with minimal water discharge needed.

\subsection{Mathematical Modeling of Power Productivity}

This section presents brief summary of the mathematical model that describes the energy dispatch problem HPP, which has been discussed in Marcelino's [5] work. Table 1 describes model parameters.

Table 1. Parameters used in model

\begin{tabular}{cl}
\hline Parameter Description \\
\hline$p h_{j t}$ & is power generated by unit $j$ at time $t$ \\
\hline$g$ & is acceleration of gravity \\
\hline$\eta_{j t}$ & is global efficiency of unit $j$ at time $t$ \\
\hline$h l_{j t}$ & is net water head of unit $j$ at time $t$ \\
\hline$q_{j t}$ & is water discharge of unit $j$ at time $t$ \\
\hline$H b_{t}$ & is hydraulic head of the reservoir \\
\hline$\Delta_{H j t}$ & is sum of pen-stock losses \\
\hline$\rho_{0 j \ldots \rho_{5 j}}$ & are coefficients obtained from the Hill Curve \\
\hline$D m$ & is requested demand $(M W)$ \\
\hline$q_{j t} m i n$ & is minimum water discharge \\
\hline$p h_{j k}^{\min }$ & is minimum power \\
\hline$q_{j t}$ max & is maximum water discharge \\
\hline$p h_{j k}^{\text {max }}$ & is maximum power \\
\hline$Z_{j k}$ & is operative zone of a generator unit \\
\hline$q_{c c}$ & is total water discharge in normal mode of operation \\
\hline
\end{tabular}

The equation which defines production of energy, in general, can be described according to Eq. (2),

$$
p h_{j t}=g \cdot \eta_{j t} \cdot h l_{j t} \cdot q_{j t} .
$$

Having that in mind and assuming that the model presented in this work is the best representation for a power plant which uses Kaplan generators, as in the case study, 
power production performed by an hydroelectric unit, in MW, can be calculated by Eq. (3),

$$
\begin{gathered}
p h_{j t}=g \cdot\left[\rho_{0 j}+\rho_{1 j} h l_{j t}+\rho_{2 j} q_{j t}+\rho_{3 j} h l_{j t} q_{j t}+\right. \\
\left.\rho_{4 j} h l_{j t}^{2}+\rho_{5 j} q_{j t}^{2}\right] \cdot\left[H b_{t}-\Delta_{H j t}\right] \cdot q_{j t} .
\end{gathered}
$$

Table 2 presents coefficients obtained by a multi-variable regression process, representing $99 \%$ of accuracy, see [5].

Table 2. Efficiency Coefficients

\begin{tabular}{|c|c|}
\hline Coefficient & Value \\
\hline$\rho_{0 j}$ & $1.4630 \mathrm{e}-01$ \\
\hline$\rho_{1 j}$ & $1.8076 \mathrm{e}-02$ \\
\hline$\rho_{2 j}$ & $5.0502 \mathrm{e}-03$ \\
\hline$\rho_{3 j}$ & $-3.5254 \mathrm{e}-05$ \\
\hline$\rho_{4 j}$ & $-1.1234 \mathrm{e}-03$ \\
\hline$\rho_{5 j}$ & $-1.4507 \mathrm{e}-05$ \\
\hline
\end{tabular}

\subsection{Multiobjective Optimization Model}

According to the mathematical model presented so far, the problem multiobjective goals are to maximize the hydroelectric productivity function (4), which is derived from the electric power function (3), and to minimize distance between NMO and OCM in function (5). The optimization variables are water flow rate of each generation unit in the following vector,

$$
x=\left[q_{1 t}, q_{2 t} \ldots q_{j t}\right] .
$$

and the bi-objective problem can be described as

$$
\begin{gathered}
\text { Maximize } F_{1}(x)=\frac{\sum_{j=1}^{J(r)} p h_{j t}}{\sum_{j=1}^{J(r)} q_{j t}}, \\
\text { Minimize } F_{2}(x)=\sqrt{\sum_{j=1}^{J(r)}\left(q_{j t}-q_{c c}\right)^{2}}
\end{gathered}
$$

subject to:

$$
\sum_{j=1}^{J(r)} p h_{j t} \cong D m, q_{j t} \min \leq q_{j t} \leq q_{j t} \max ,
$$




$$
\begin{gathered}
p h_{j k}^{\min } \sum_{k=1}^{\emptyset_{j}} Z_{j k} \leq p h_{j t} \leq p h_{j k}^{\max } \sum_{k=1}^{\emptyset_{j}} Z_{j k}, \\
Z_{j k} \in\{0,1\}, \sum_{k=1}^{\emptyset_{j}} Z_{j k} \leq 1 .
\end{gathered}
$$

The first objective function determines how much power the plant is able to produce with a given volume of water. Maximizing this function means to produce more power using less water. The numerator of $F_{1}$ is the production function: as this number increases, objective function value also grows. When the denominator of $F_{1}$ is decreased, productivity ratio is also reduced. This function is subject to operational constraints, i.e., the sum of all generation units production must be equal to total power demand required to be produced by the HPP. Power production must also comply with generation units operational limits, represented by inequality constraints of objective function.

The second objective function, $F_{2}$, measures distance between water discharge used in NMO and water discharge used in OCM. This function was proposed because, in practice, the plant technical operation staff has the predilection for using NMO. This function shows that there are operation points in OCM which are closer to NMO but still ensure the maximization of energy production efficiency. This contributes to a new culture development by the HPP operation staff, increasing their confidence on OCM.

The first constraint indicates that power to be delivered should be equal to power requested to be produced by the HPP. The second constraint states that calculated flow rate must comply with the minimum and maximum flow capacity of each generation unit. The third constraint requires that the corresponding generated power must comply with the minimum and maximum power capacity of each generation unit. At last, the fourth constraint ensures that each generation unit maintains its operating status, i.e. it stays ON or OFF during the whole production period.

\section{Experiments}

As a computational simulation test, this paper proposes as test scenario a HPP in Brazil with nominal installed capacity of $396 M W$. The HPP has in its powerhouse 6 power generators. Water discharge varies between $[70,140] \mathrm{m}^{3} / \mathrm{s}$ and power generators are operating in [35,66] $M W$ range. The plant value of $H b$ ranges between [32,56] $\mathrm{m}$. All generation units are considered identical, so Hill Curve coefficients are the same for each unit. Two experiments are presented to validate the adopted multiobjective approach.

The first experiment aims to compare and to analyse the value found in monoobjective approach proposed by Marcelino [5] (the solution was found via a Differential Evolution algorithm [14]) and results found in proposed multiobjective approach. The second experiment aims to assess Pareto front quality obtained by NSGA-II and SPEAII algorithms. Initialization parameters of both multiobjective algorithms are: population size (50 individuals), crossover probability (80\%), mutation probability (2\%) and iterations (50 generations). 


\subsection{Experiment 1}

The main goal of this experiment is to verify if the mono-objective solution presented in [5] is a reasonable solution for the multiobjective approach solved via NSGA-II and SPEA-II. For that, a demand of $320 M W$ is established, since this is a typical demand of the HPP. The reservoir hydraulic head, $\mathrm{Hb}$ is set to $54 \mathrm{~m}$.
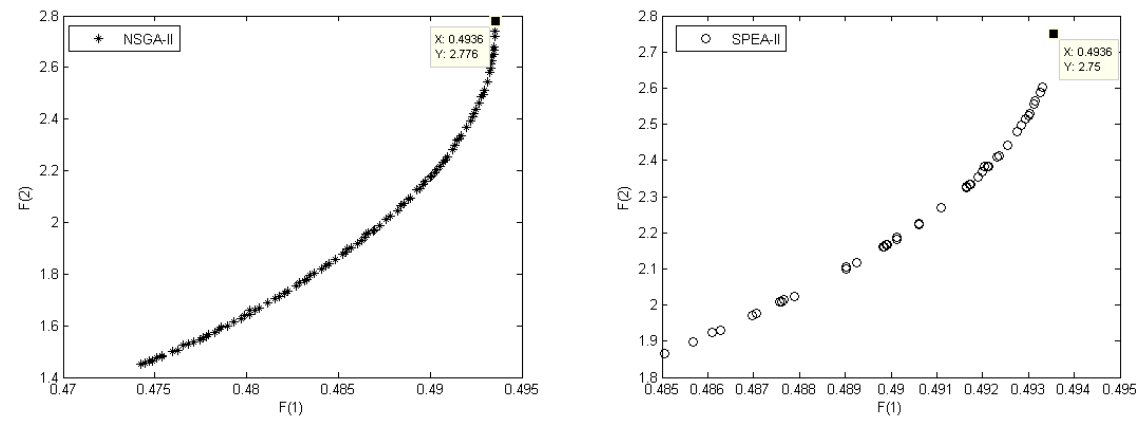

Fig. 3. Pareto Front to $320 \mathrm{MW}$

Fig. 3 shows that there is a point, belonging to Pareto Front obtained by NSGA-II and SPEA-II, which is very close to the result reported by DE/best/1/bin [5].

A simulation report for DE/best/1/bin, NSGA-II and SPEA-II is shown in Table 3. This table presents results for the best individual obtained by total water flow $\left(q_{j t}\right)$ and, using these values, other parameters are calculated from the mathematical model.

As a comparison to multiobjective optimization results, resulting power production per unit would be $53.33 M W$ per unit if NMO is used, which corresponds to a global water flow rate of $655.05 \mathrm{~m}^{3} / \mathrm{s}$. In this context, the productivity of NMO for this experiment is 0.48 . The value found by $\mathrm{DE}$ algorithm after maximizing productivity is 0.4906 (DE/best/1/bin). Thus, DE/best/1/bin configuration achieves higher productivity rate than NMO and consequently higher economy of water discharge, corresponding to a water flow rate of $2.54 \mathrm{~m}^{3} / \mathrm{s}$. For the solution point obtained using NSGA-II, water flow economy is even better with $6.14 \mathrm{~m}^{3} / \mathrm{s}$ whereas water flow rate is $5.66 \mathrm{~m}^{3} / \mathrm{s}$ for case of SPEA-II. Expanding to one month, this is equivalent to saving approximately 6.5 million $\mathrm{m}^{3}$ of water using mono-objective approach, 14.4 million $\mathrm{m}^{3}$ of water using the solution of SPEA-II and 15.7 million $\mathrm{m}^{3}$ of water using the solution of NSGA-II algorithm.

According to [15], monthly water consumption for a city of 300,000 inhabitants is, on average, 1.1 million $\mathrm{m}^{3}$. Belo Horizonte, which is the 6th biggest Brazilian city, has a population of 2.4 million habitants. In a simple analogy, 15.7 million $\mathrm{m}^{3}$ is sufficient to supply the city of Belo Horizonte for almost 2 months, on average. It is also easy to check that, in OCM operation, all units reached maximum efficiency of $93 \%$ by using determined water flow rate. 
Table 3. General Simulation Report for a Power Demand of 320 MW

\begin{tabular}{|c|c|c|c|c|c|}
\hline \multicolumn{6}{|c|}{ Mono-objective algorithm: DE/best/1/bin - $(\mathrm{Hb})=54 m-$ [5] } \\
\hline UN & $p h_{j t}(M W)$ & $q_{j t}\left(\mathrm{~m}^{3} / \mathrm{s}\right)$ & $\eta_{j t}(\%)$ & $h l_{j t}(m)$ & $\Delta_{H j t}(m)$ \\
\hline 1 & 48,763 & 99,441 & 0,93 & 53,804 & 0,19595 \\
\hline 2 & 54,589 & 111,26 & 0,93 & 53,836 & 0,16354 \\
\hline 3 & 55,81 & 113,74 & 0,93 & 53,839 & 0,16103 \\
\hline 4 & 56,429 & 115,00 & 0,93 & 53,841 & 0,15925 \\
\hline 5 & 53,122 & 108,26 & 0,93 & 53,841 & 0,15925 \\
\hline 6 & 51,438 & 104,83 & 0,93 & 53,839 & 0,16103 \\
\hline SUM & 320,15 & 652,51 & \multicolumn{3}{|c|}{ Flow in SCM: 655,05 $\left(\mathrm{m}^{3} / \mathrm{s}\right)$} \\
\hline DIF & $+0,15$ & 2,54 & \multicolumn{3}{|c|}{ Productivity index: 0.4906} \\
\hline \multicolumn{6}{|c|}{ Multiobjective algorithm: NSGA-II $-(H b)=54 m$} \\
\hline UN & $p h_{j t}(M W)$ & $q_{j t}\left(m^{3} / s\right)$ & $\eta_{j t}(\%)$ & $h l_{j t}(m)$ & $\Delta_{H j t} \quad(m)$ \\
\hline 1 & 53,475 & 108,274 & 0,93 & 53,759 & 0,24149 \\
\hline 2 & 54,229 & 109,805 & 0,93 & 53,798 & 0,20155 \\
\hline 3 & 53,363 & 108,047 & 0,93 & 53,802 & 0,19845 \\
\hline 4 & 53,325 & 107,971 & 0,93 & 53,804 & 0,19625 \\
\hline 5 & 52,891 & 106,094 & 0,93 & 53,804 & 0,19625 \\
\hline 6 & 53,182 & 108,110 & 0,93 & 53,802 & 0,19845 \\
\hline SUM & 320,467 & 648,903 & \multicolumn{3}{|c|}{ Flow in SCM: $\mathbf{6 5 5 , 0 5}\left(\mathrm{m}^{3} / \mathrm{s}\right)$} \\
\hline DIF & $+0,46$ & 6,14 & \\
\hline \multicolumn{6}{|c|}{ Multiobjective algorithm: SPEA-II $-(H b)=54 m$} \\
\hline UN & $p h_{j t}(M W)$ & $q_{j t}\left(m^{3} / s\right)$ & $\eta_{j t}(\%)$ & $h l_{j t}(m)$ & $\Delta_{H j t} \quad(m)$ \\
\hline 1 & 52,221 & 105,747 & 0,93 & 53,796 & 0,20377 \\
\hline 2 & 51,751 & 104,804 & 0,93 & 53,83 & 0,17007 \\
\hline 3 & 51,976 & 105,255 & 0,93 & 53,833 & 0,16745 \\
\hline 4 & $\begin{array}{l}5,687 \\
\end{array}$ & 112,787 & 0,93 & 53,834 & 0,1656 \\
\hline 5 & 54,457 & 110,269 & 0,93 & 53,834 & 0,1656 \\
\hline 6 & 54,358 & 110,526 & 0,93 & 53,833 & 0,16745 \\
\hline SUM & 320,452 & 649,390 & \multirow{2}{*}{\multicolumn{3}{|c|}{$\begin{array}{l}\text { Flow in SCM: } \mathbf{6 5 5 , 0 5}\left(\mathrm{m}^{3} / \mathrm{s}\right) \\
\text { Productivity index: } \mathbf{0 . 4 9 3 5}\end{array}$}} \\
\hline DIF & $+0,45$ & 5,66 & & & \\
\hline
\end{tabular}

\subsection{Experiment 2}

In this experiment, NSGA-II and SPEA-II are executed for 30 times each, and a combined Pareto Front is generated for each algorithm. After that, a dominance routine is applied to generate the final Pareto Front for power demand of $320 M W$. Fig. 4 shows the final Pareto Front for NSGA-II and SPEA-II.

Note that the multiobjective approach presents several solutions for the problem. The Pareto optimal set indicates different solutions among NMO (characterized as the lowest point of the Pareto Front) and OCM (other points on Pareto front). The solution set has an important role in operational scope since the HPP operation team can realize the OCM is not far, in terms of water discharge, from NMO. In this way, it is shown that OCM is a type of control that can be used without harming departing from current operational practises. This leads to optimization technique usage acceptance in industrial environment. 


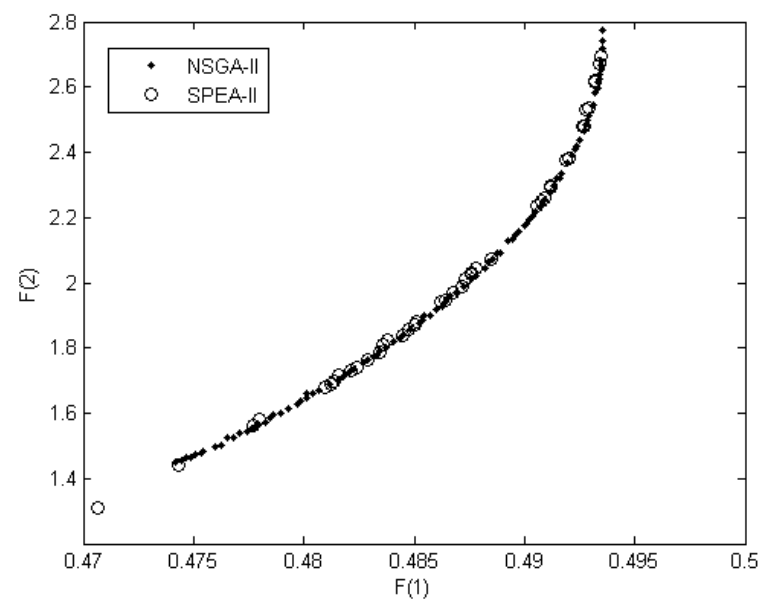

Fig. 4. Comparison between combined Pareto Fronts

\section{Statistical Analysis}

\subsection{ANOVA}

Analysis of variance (ANOVA) is a statistical technique that evaluates hypotheses about several populations means and variances. This analysis evaluates primarily if there is a significant difference between the mean and the influence factors on some dependent variable. In this way, ANOVA is used when one wants to decide if sample differences are real (i.e., caused by significant differences in observed populations) or casual (resulting from mere sampling variability) [16]. Therefore, this analysis assumes that chance only produces small deviations, the major differences being generated by real causes. The null and alternative hypotheses to be tested by ANOVA here are:

- Null hypothesis $H_{0}$ : populations means are equal;

- Alternative hypothesis $H_{1}$ : populations means are different, i.e. , at least one of the means is different from the others.

To perform ANOVA hypothesis test, previously tested power demand $(320 \mathrm{MW})$ is used in 30 runs of NSGA-II and SPEA-II algorithms. For each run, the obtained Pareto Front quality is assessed using S-Metric, and the mean value of S-Metric is obtained for each result. S-Metric is a commonly accepted quality measure for comparing approximations of Pareto fronts generated by multiobjective optimizers [12]. This metric calculates hypervolume of a multi-dimensional region enclosed by $\beta$ and a reference point, thus calculating the region extent that $\beta$ dominates. Table 4 shows results of the performed ANOVA, indicating that the hypothesis of equality between S-Metric means is rejected with statistical significance of $95 \%$, because P-Value is equal to 0.0285 , meaning that at least the S-Metric mean value of NSGA-II and SPEA-II algorithms. 
Table 4. Reports by Analysis of variance

\begin{tabular}{cccccc}
\hline \multicolumn{5}{c}{ ANOVA } \\
\hline Source & SS & dF & MS & F & Prob $>$ F \\
\hline Columns & 0.01251 & 1 & 0.01251 & 5.04 & $\mathbf{0 . 0 2 8 5}$ \\
Error & 0.14391 & 58 & 0.00248 & & \\
Total & 0.15643 & 59 & & & \\
\hline
\end{tabular}

Despite the indication of ANOVA that there is a significant difference between algorithms, it is not possible to say which algorithm is the best one. Two tests, Tukey and Permutation tests, are used to determine what the difference exists.

\subsection{Tukey and Permutation tests}

Given its ability to analyse multiple data sets, this study used ANOVA with Tukey test to find some information that differentiates the algorithms mentioned above. This statistical method can be interpreted as a comparison of means between different groups, with variance between all individuals within those groups. Tukey's strategy is to define the least significant difference between means. The hypothesis to be considered in this test is the equality of data sets series results and adopted a confidence interval of $95 \%$ [17]. Permutation tests are non-parametric statistical methods which estimate a reference distribution by calculating all possible values (or at least a considerably large set) of a test statistic under rearrangements of labels on a set of observed data points [17]. Fig. 5 and Fig. 6 shows results of the performed Tukey and Permutation tests.

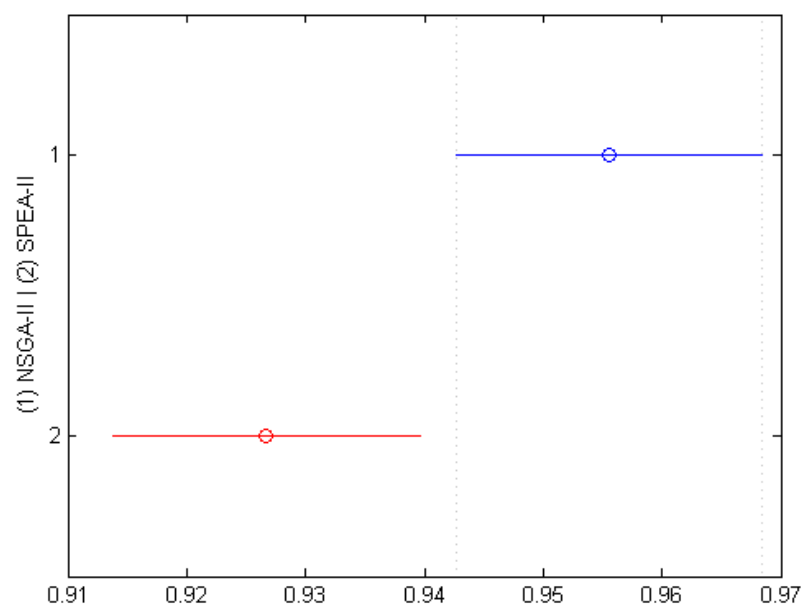

Fig. 5. Left: Tukey test results 


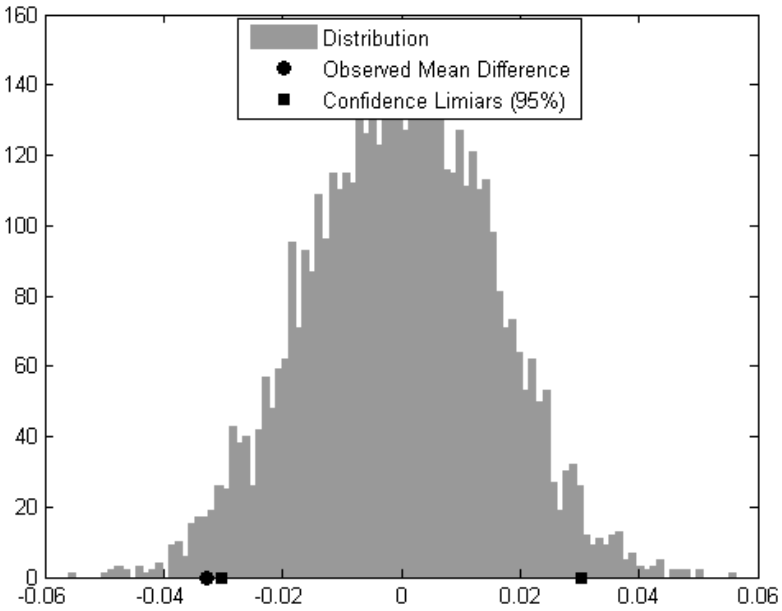

Fig. 6. Permutation test results

Tukey test shows that there is a difference between sets of data tested indicating that, on the mean, the NSGA-II algorithm has higher value of S-Metric with $95 \%$ confidence. Permutation test confirms this information by the fact that the average value represented by the observed mean difference ("black ball") is outside the confidence interval tested, indicating that the data sets are different. This proves that NSGA-II solves the multiobjective energy dispatch of HPP problem better when compared to SPEA-II. It is worthwhile to notice that both NSGA-II and SPEA-II showed superior results to those found in mono-objective approach using DE algorithm, as shown in Table 3.

\section{Conclusion}

This paper presented a multiobjective approach to solve the energy dispatch problem of Hydroelectric Power Plants using NSGA-II and SPEA-II algorithms. Results of practical experiments indicate that it is possible to identify operating points near NMO that present high productive efficiency. In one experiment, a selected point in Pareto Front with power demand of $320 \mathrm{MW}$ showed a productivity index equal to 0.4936 . This point is very similar, in both objective functions, to results found in previous mono-objective approaches, granting reliability to the results and indicating a saving in energy production of 15.7 million $\mathrm{m}^{3}$ of water using NSGA-II. This amount of water is able to supply a city of 2.4 million people for 2 months. Through statistical inference, it was possible to see that NSGA-II algorithm is shown to have greater robustness than SPEAII algorithm to solve this problem. To conclude, it is important to mention that OCM approach can be easily adapted to run inside other kinds of plants, similar to HPP case study discussed here, as it is a generalist approach. 


\section{Acknowledgement}

The authors would like to thank CEMIG and ORTENG, for the cession of operational data and support, CEMIG and ANEEL for the grant GT333/2011, CEFET-MG and INESC-Tec for the infrastructure used for this project, and CAPES, CNPq and FAPEMIG for financial support.

\section{References}

1. P. Pezzini, O. Gomis-Bellmunta, and A. Sudrià-Andreua. Optimization techniques to improve energy efficiency in power systems. ELSEVIER In: Renewable and Sustainable Energy Reviews, 15:2028 - 2041, 2011.

2. R. Baños and et al. Optimization methods applied to renewable and sustainable energy: A review. ELSEVIER, In: Renewable and Sustainable Energy Reviews, 15:1753 - 1766, 2011.

3. E. Finardi and E. L. da Silva. Unit commitment of single hydroelectric plant. Electric Power System Research, 75:116-123, 2005.

4. P. L. Abrao, E. Wanner, and P. Almeida. A novel movable partitions approach with neural networks and evolutionary algorithms for solving the hydroelectric unit commitment problem. Proceeding of the 15h annual conference on GECCO, pages 1205-1212, 2013.

5. C. Marcelino, E. Wanner, and P. Almeida. A novel mathematical modeling approach to the electric dispatch problem: Case study using differential evolution algorithms. Proceedings of conference: IEEE Congress on Evolutionary Computation (CEC), pages 400-407, 2013.

6. M. A. Abido. Multiobjective evolutionary algorithms for electric power dispatch problem. IEEE Transactions on Evolutionary Computation, VOL. 10, NO. 3:315-319, June, 2006.

7. B. Zhou, K.W. Chan, T. Yu, and C. Y. Chung. Equilibrium-inspired multiple group search optimization with synergistic learning for multiobjective electric power dispatch. IEEE Transactions on Power Systems, VOL. 28, NO. 4: 3534-3545 , November., 2013.

8. C. M. Fonseca and P. J. Fleming. Genetic algorithms for multiobjective optimization: Formulation, discussion and generalization. In S. Forrest (Ed.), Proceedings of the Fifth International Conference on Genetic Algorithms, San Mateo, California, pp. 416-423. Morgan Kaufmann., 1993.

9. J. Horn, N. Nafpliotis, and D. E. Goldberg. A niched pareto genetic algorithm for multiobjective optimization. In Proceedings of the First IEEE Conference on Evolutionary Computation, IEEE World Congress on Computational Computation, Volume 1, Piscataway, NJ, pp. 82-87. IEEE Press., 1994.

10. N. Srinivas and K. Deb. Multiobjective optimization using nondominated sorting in genetic algorithms. Evolutionary Computation 2(3), 221-248, 1994.

11. K. Deb, S. Agrawal, A. Pratap, and T. Meyarivan. A fast elitist nondominated sorting genetic algorithm for multi-objective optimization: Nsga-ii. In M. S. et al. (Ed.), Parallel Problem Solving from Nature - PPSN VI, Berlin, pp. 849-858. Springer., 2000.

12. E. Zitzler. Evolutionary algorithms for multiobjective optimization: Methods and applications. PhD thesis, Swiss Federal Institute of Technology (ETH), Zurich,, 1999.

13. E. Zitzler and L. Laumanns, M. Thiele. Spea2: Improving the strength pareto evolutionary algorithm. TIK-Report 103, May, 2001.

14. R. Storn and K. Price. Differential evolution: a simple and efficient adaptative scheme for global optimization over continuous spaces. Techinical report TR-95-012, ICSI, Berkley, 1995.

15. G. Carneiro and J. Chaves. Pilot study to establish the flow of comfort for residential water consumption in the city of ponta grossa. In 4th Meeting of the general engineering and technology fields. Brazil., 2008. 
16. D. Montgomery and G. Runger. Applied statistics and probability for engineers. 4th ed. Rio de Janeiro, 2009.

17. E.G. Carrano, E.F. Wanner, and R. Takarashi. A multicriteria statistical based comparison methodology for evaluating evolutionary algorithms. IEEE Transactions on Evolutionary Computation, 15:848 - 870, 2010. 